\title{
Circadian Changes of Dendritic Spine Geometry in Mouse Barrel Cortex
}

\author{
Malgorzata Jasinska1*, Olga Woznicka², Ewa Jasek-Gajda', Grzegorz J. Lis", \\ Elzbieta Pyza² and Jan A. Litwin ${ }^{1}$
}

${ }^{1}$ Department of Histology, Jagiellonian University Medical College, Krakow, Poland, ${ }^{2}$ Department of Cell Biology and Imaging, Institute of Zoology and Biomedical Research, Jagiellonian University, Krakow, Poland

\section{OPEN ACCESS}

Edited by:

Marcos G. Frank, Washington State University Health Sciences Spokane, United States

Reviewed by:

Dante Picchioni,

National Institutes of Health (NIH),

United States

Julie Seibt,

University of Surrey, United Kingdom

${ }^{*}$ Correspondence:

Malgorzata Jasinska

malgorzata.m.jasinska@uj.edu.pl

Specialty section:

This article was submitted to Sleep and Circadian Rhythms, a section of the journal

Frontiers in Neuroscience

Received: 01 July 2020

Accepted: 26 August 2020

Published: 29 September 2020

Citation:

Jasinska M, Woznicka O,

Jasek-Gajda E, Lis GJ, Pyza E and

Litwin JA (2020) Circadian Changes

of Dendritic Spine Geometry in Mouse

Barrel Cortex.

Front. Neurosci. 14:578881.

doi: 10.3389/fnins.2020.578881
The circadian rhythmicity changes the density and shape of dendritic spines in mouse somatosensory barrel cortex, influencing their stability and maturation. In this study, we analyzed the main geometric parameters of dendritic spines reflecting the strength of synapses located on these spines under light/dark (12:12) and constant darkness conditions, in order to distinguish between endogenously regulated and lightdriven parameters. Using morphological analysis of serial electron micrographs, as well as three-dimensional reconstructions, we found that the light induces elongation of single-synapse spine necks and increases in the diameter of double-synapse spine necks, increasing and decreasing the isolation of synapses from the parent dendrite, respectively. During the subjective night of constant darkness, we observed an enlargement of postsynaptic density area in inhibitory synapses and an increase in the number of polyribosomes inside double-synapse spines. The results show that both endogenous effect (circadian clock/locomotor activity) and light affect the morphological parameters of single- and double-synapse spines in the somatosensory cortex: light reduces the efficiency of excitatory synapses on single-synapse spines, increases the effect of synaptic transmission in double-synapse spines, and additionally masks the endogenous clock-driven enlargement of inhibitory synapses located on double-synapse spines. This indicates a special role of double-synapse spines and their inhibitory synapses in the regulation of synaptic transmission during both circadian and diurnal cycles in the mouse somatosensory cortex.

Keywords: circadian rhythmicity, influence of light, dendritic spine morphology, neural plasticity, electron microscopy, somatosensory cortex

\section{INTRODUCTION}

The quantitative and qualitative changes of small dendritic protrusions-dendritic spinesassociated with activity-dependent neural plasticity have been observed in various areas of the brain. The importance of such changes results from the general belief that increase/decrease in the number and size of dendritic spines is associated with memory formation processes (Kasai et al., 2010). The morphological modifications of spines could be even more significant than their numerical changes (Geinisman et al., 2000, 2001), because they provide an observable reflection of spine function (Yuste et al., 2000). The shape of dendritic spines is highly flexible and can 
be modified in a relatively short time by experience-dependent synaptic plasticity: long-term depression, long-term potentiation (Nikonenko et al., 2002; Bourne and Harris, 2008; Bosch and Hayashi, 2012), or associative learning process (Leuner and Shors, 2004; Bourne and Harris, 2008; Kasai et al., 2010; Jasinska et al., 2016).

The density and morphology of spines are additionally dependent on the activity of animal during the 24-h period (sleep/wakefulness) (Maret et al., 2011; Yang and Gan, 2012; Acosta-Peña et al., 2015; Havekes et al., 2016) or on the phase of the circadian cycle (day/night) (Ikeno et al., 2013; Liston et al., 2013; Jasinska et al., 2015, 2019). While studies of the geometric parameters of the spines during sleep/wake cycle are more frequent (Frank and Cantera, 2014; van der Zee, 2015; Areal et al., 2017; Raven et al., 2018), the information on morphological changes of the spines in the circadian rhythm is relatively scarce. Investigation of such changes seems to be important, because circadian rhythmicity affects synaptic plasticity irrespective of sleep/wake state (Frank and Cantera, 2014).

Circadian neural plasticity has been extensively studied in insects and focused on the visual system, as well as the motor neurons (Frank, 2016; Krzeptowski et al., 2018). The diurnal and circadian changes were found in various synapses (Pyza and Meinertzhagen, 1993; Górska-Andrzejak et al., 2013; Ruiz et al., 2013; Woźnicka et al., 2015), as well as in the size and morphology of axons, dendrites, or even whole neurons (Pyza and Meinertzhagen, 1999; Górska-Andrzejak et al., 2005; Mehnert et al., 2007; Fernández et al., 2008).

In rodents, cyclic morphological modifications of synapses and neurons were observed in the superchiasmatic nucleus-a structure directly responsible for synchronization of the circadian rhythmicity in the body (Becquet et al., 2008; Girardet et al., 2010), as well as in the retina (Behrens et al., 1998; Balkema et al., 2001). The studies of dendritic spines related to diurnal or circadian plasticity are limited to the hippocampus and some areas of the neocortex. It has been shown that under light/dark (LD) conditions dendritic spines are more numerous during the active phase of animals in rat hippocampus (Ikeda et al., 2015) and infralimbic cortex (Perez-Cruz et al., 2009b), as well as in mouse motor cortex (Liston et al., 2013). It seems that circadian oscillations of glucocorticoids affect the dynamics of spines in the motor and somatosensory cortex (Liston and Gan, 2011; Liston et al., 2013). The peak of glucocorticoid release occurs at the beginning of the active phase of animals (Cheifetz, 1971; Chung et al., 2011), when intensified dendritic spine formation is also observed (Liston and Gan, 2011).

In the mouse barrel cortex, the density of spines with single excitatory synapses (single-synapse spines) increases in the day under LD conditions, whereas the number of double-synapse spines is higher during the night/subjective night (LD/constant darkness, DD), what indicates light-dependent regulation of single-synapse spine density and endogenous regulation of the number of double-synapse spines (Jasinska et al., 2015). The increase in the density of single- and double-synapse spines is accompanied by a simultaneous increase in the number of excitatory and inhibitory synapses, respectively (Jasinska et al., 2014; Jasinska et al., 2015).
Diurnal changes in the density of dendritic spines appear to be closely related to the photoperiod (long and short day) and brain subregion (CA1/DG hippocampus; Ikeno et al., 2013) and can even fluctuate between the hemispheres of the brain (left/right; Perez-Cruz et al., 2009a).

In the mouse barrel cortex, the shape modifications of singlesynapse spines are driven by the circadian clock, whereas in double-synapse spines they remain under the influence of the light (Jasinska et al., 2019).

The light also promotes enlargement and maturation of both single- and double-synapse spines. In the rest phase, an increase in the number of mushroom single-synapse spines was observed under LD conditions in mouse somatosensory cortex, whereas double-synapse spines contained spine apparatus (SA) indicating their maturity (Jasinska et al., 2019). In hippocampal CA1 field, the number of spines with large heads increased at the beginning of the active phase (Ikeda et al., 2015).

Dendritic spine is usually described as a structure with a distinct head and neck, although the degree of head/neck distinction is variable and depends on the overall shape of the spine (Peters and Kaiserman-Abramof, 1970; Bourne and Harris, 2007). It is widely believed that the head is functionally more important than the neck, probably due to the fact that the majority of excitatory synapses are located on the spine heads (Maiti et al., 2015). This rule, however, is not fully justified in case of spines with two different synapses (double-synapse spines), located in mouse somatosensory cortex, in which the excitatory synapses are usually located on the spine heads, while the inhibitory synapses on the spine necks (Jasinska et al., 2006).

Dendritic spines are separate biochemical compartments allowing control of protein flow, compartmentalization of calcium (Denk et al., 1995; Kovalchuk et al., 2000; Noguchi et al., 2005), and regulation of second messenger diffusion between the head and the parent dendrite (Tønnesen and Nägerl, 2016). The general morphology of a spine in which wide head is connected to the dendrite via narrow neck allows complete isolation of the spine from dendrite shaft or at least slows down diffusion between spine head and the shaft (Sorra and Harris, 2000; Bloodgood and Sabatini, 2005). Because of the barrier created by the spine neck, the concentration of calcium in the head can reach a higher level than in the parent dendrite (Korkotian and Segal, 2007; Segal, 2010), what might be an effective way of protecting the neuron from overstimulation or excitotoxicity (Sorra and Harris, 2000; Segal, 2010).

The spine necks can also electrically separate the spines (Araya et al., 2014) by isolating the inputs of synapses located on adjacent spines, as well as isolating them from dendrite (Araya et al., 2006). It suggests that spine neck might play a significant role in the regulation of synapse effectiveness.

Length and diameter of spine neck are important for neck resistance (Wickens, 1988; Spacek and Harris, 1997). Interestingly, no correlation has been found between these parameters, and it seems that they are regulated independently (Arellano et al., 2007). The increase in the spine neck length reduces the strength of the synapse (Araya et al., 2014). It has been shown that excitatory postsynaptic potential amplitudes are inversely proportional to spine neck length, as well as that 
spines with long necks have a weak or negligible contribution to somatic voltage, but after synaptic stimulation, they could shorten the necks and increase synaptic efficiency (Araya et al., 2014). Parameters of the spine neck (Fifkova and Anderson, 1981; Jasinska et al., 2006; Tønnesen et al., 2014).

The earlier studies of the circadian rhythm of spine morphology were focused on spine shapes, which indicate the level of spine stability, but do not provide information about the geometric parameters of the spines, which indirectly influence the strength and efficiency of spine-associated synapses. In our previous study, we analyzed the influence of the circadian clock/locomotor activity and light on the shape of spines (stubby, thin, and mushroom) and their content [smooth endoplasmic reticulum (sER), SA] reflecting the level of spine maturity and stability. In the present exploratory study, we investigate circadian changes of measurable geometric parameters (spine length and volume, spine head diameter, spine neck length, and diameter), as well as postsynaptic density (PSD) area indicative of synapse strength and the number of polyribosomes reflecting the local protein synthesis, which plays a key role in the modification of synapses located on the spines.

\section{MATERIALS AND METHODS}

We examined the same collection of ultrathin sections, which was used in our previous study (Jasinska et al., 2019).

\section{Animals}

The experiments were performed on C57BL male mice aged 5 to 6 weeks. This study was carried out in accordance with the Council Directive 2010/63EU of the European Parliament and the Council of 22 September 2010 on the protection of animals used for scientific purposes and approved by the Animal Care and Use Committees of the Jagiellonian University.

\section{Analysis of Locomotor Activity}

All animal were kept for 2 weeks under the following conditions: LD $12: 12$ (12 h of light and $12 \mathrm{~h}$ of dark), light $60 \mathrm{~lx}, 25^{\circ} \mathrm{C}$, and $50 \%$ humidity to get used to standard light conditions. Next, the mice were divided into LD $(n=8)$ and DD $(n=8)$ groups. The mice in the LD group were kept for the next 2 weeks under LD 12:12 and in the DD group-under constant darkness. The animals were fed a standard diet and water ad libitum.

From the beginning of experiments, mice stayed in the cages with free access to a running wheel coupled with a 16-channel electromagnetic pulse counter (MIKI 1; Autel, Poland). The running activity was continuously recorded: the number of wheel rotations per minute was counted and then transferred to PC computer by RS232 interface. Data were recorded and saved on a computer disk by using RealTerm software (RealTerm: Serial/TCP Terminal $2 \cdot 0 \cdot 0 \cdot 70^{1}$ ). The obtained data were analyzed using NIH ImageJ ActogramJ software ${ }^{2}$.

${ }^{1}$ realterm.sf.net

${ }^{2}$ http://rsb.info.nih.gov/ij/
All mice showing locomotor rhythmicity under LD conditions were selected for further experiments. Eight mice were killed $2 \mathrm{~h}$ after the beginning of the light phase or the subjective day $(n=4$ per subgroup LD REST and DD REST), and eight mice were killed $2 \mathrm{~h}$ after the beginning of the dark phase or the subjective night ( $n=4$ per subgroups LD ACTIVE and DD ACTIVE).

\section{Transmission Electron Microscopy}

The mice were anesthetized with Morbital (25-30 mg/kg of body weight; Biowet, Pulawy, Poland) and perfused through the heart with $20 \mathrm{~mL}$ of rinse buffer $(0.2 \%$ glutaraldehyde and $2 \%$ paraformaldehyde in $0.1 \mathrm{M}$ phosphate buffer, $\mathrm{pH} 7.4$ ), followed by 100 to $150 \mathrm{~mL}$ of fixative $(2.5 \%$ glutaraldehyde and $2 \%$ paraformaldehyde in $0.1 \mathrm{M}$ phosphate buffer, $\mathrm{pH} 7.4$ ). Immediately after perfusion, the brains were removed and kept in the same fixative for $24 \mathrm{~h}$ at $4^{\circ} \mathrm{C}$.

Next, after a wash in $0.1 \mathrm{M}$ phosphate buffer ( $\mathrm{pH} 7.4$ ), $60 \mu \mathrm{m}$ tangential vibratome sections were cut from the barrel cortex region and examined under a stereomicroscope (Nikon Optiphot, Japan). Only sections containing the barrel field cortex were collected for further processing. The sections were washed in $0.1 \mathrm{M}$ cacodylate buffer ( $\mathrm{pH} 7.4$ ), postfixed twice with $1 \%$ osmium tetroxide in $0.1 \mathrm{M}$ cacodylate buffer, $\mathrm{pH}$ 7.4 (the first change containing $1.5 \%$ potassium ferrocyanide), washed in $70 \%$ ethanol containing $1 \%$ uranyl acetate, and after dehydration in graded series of ethanol, embedded in Epon (Polysciences, United States) between two siliconcoated glass slides.

The region of $\mathrm{B} 2$ barrel was identified according to the procedure described previously (Jasinska et al., 2010). The embedded slices were trimmed into blocks, and a series of 10 to 15 successive ultrathin sections (65-75 nm thick) were cut from each sample. The sections were collected on formvar-coated copper-palladium slot grids and contrasted with $1 \%$ lead citrate.

For examination of dendritic spine morphology, three to five series of electron micrographs (10-15 serial micrographs each) of the B2 barrel central area in which cell bodies are sparse were taken from each mouse at $30 \mathrm{~K}$ magnification in JEOL JEM 2100 transmission electron microscope (JEOL, Japan). The micrographs were aligned using Adobe Photoshop CS software, and stacks of serial images were prepared.

\section{Morphological Analysis of Single- and Double-Synapse Spines}

Only spines that were completely contained within each volume sample were selected for the quantitative analysis. Images of 201 single-synapse spines and 89 double-synapse spines from both LD and DD groups were chosen for morphological measurements and three-dimensional (3D) reconstruction (single synapse-spines-LD REST: 42, LD ACTIVE: 37, DD REST: 51, DD ACTIVE: 71; double synapse-spines-LD REST: 18, LD ACTIVE: 25, DD REST: 20, DD ACTIVE: 26).

Dendritic spines were defined according to Knott et al. (2002). Synapses were characterized according to Jasinska et al. (2010, 2015). The distinction between excitatory and inhibitory synapses 

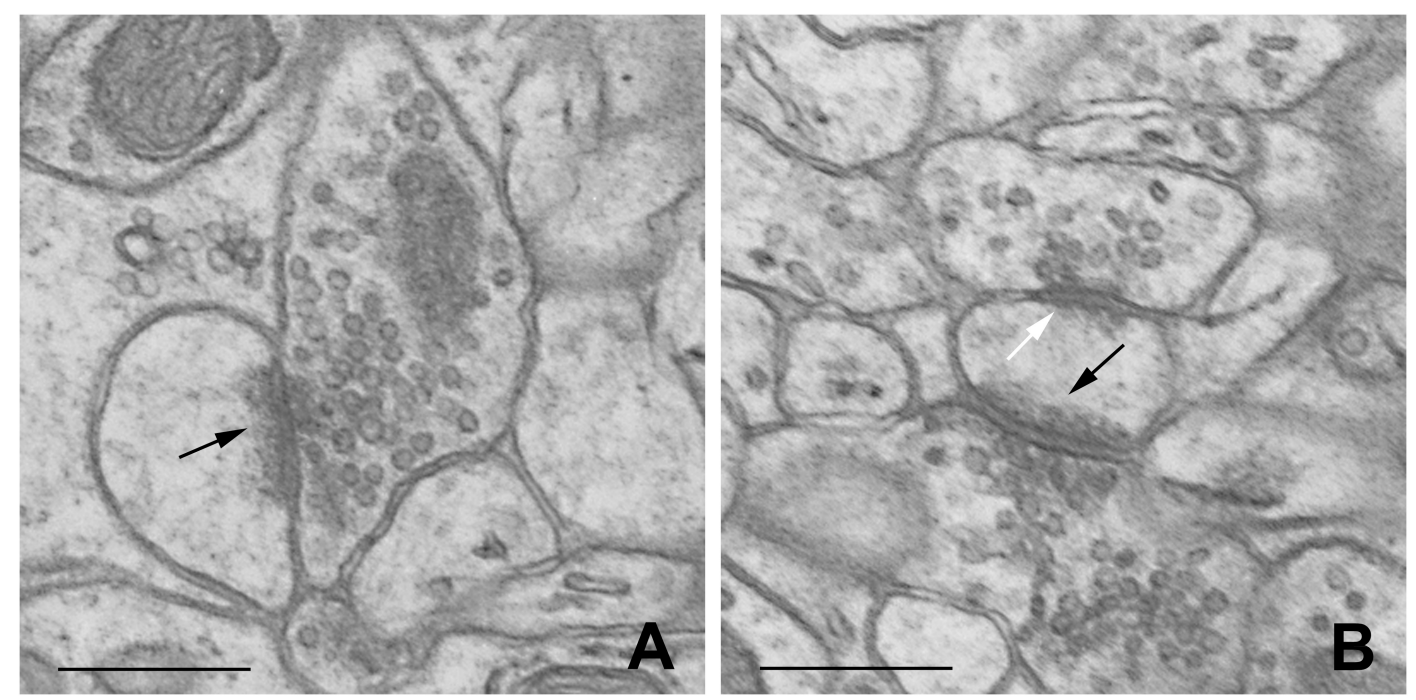

FIGURE 1 | Representative electron micrographs of B2 barrel hollow showing single-synapse spine (A) and double-synapse spine (B). Excitatory and inhibitory synapses are indicated by black and white arrows, respectively. Scale bars: $0.5 \mu \mathrm{m}$.

was based on the symmetry of synaptic membranes and on the appearance of synaptic vesicles (Figure 1).

$3 \mathrm{D}$ reconstructions of the spines were performed using $3 \mathrm{D}$ Studio Max software (Discreet Logic, Montreal, QC, Canada) (Jasinska et al., 2016).

The length of the spine and of its neck was measured after 3D reconstruction of the spine (Jasinska et al., 2016; Figure 2). In the electron micrographs, total spine area and the areas of spine head and neck were measured, and on the basis of areas in serial sections and according to the known thickness of the sections, volumes of the structures were calculated. The diameter of spine head was measured as the longest diameter parallel to PSD (Bourne and Harris, 2011). Three measurements of neck diameter at different levels were made and averaged (Arellano et al., 2007; Jasinska et al., 2016). The areas of PSD were calculated according to Ostroff et al. (2002). The polyribosomes were counted in each spine, and their number per spine was assessed (Jasinska et al., 2013, 2016). Using 3D reconstructions, distribution of polyribosomes in the spines (location in the head or neck) was estimated.

All measurements were performed using NIH ImageJ software (Analyze-Measure, Cell Counter Plugin; see text footnote 2).

The counting and measurements were done blind-the observer did not know whether the micrographs were taken from LD or DD and ACTIVE or REST groups.

\section{Statistical Analysis}

All data were analyzed using GraphPad Prism 5.01 software (GraphPad Software Inc., United States).

To compare the combined effect of animals' activity and light conditions on all morphological measurements (total spine volume, the volume of spine head and neck, the length of spine and spine neck, the diameter of spine head and neck, the area of PSD, the number of polyribosomes), 2-way analysis of variance (ANOVA) with post hoc Bonferroni test preceded by Kolmogorov-Smirnov normality test was used. Differences in the location of polyribosomes in dendritic spines between REST and ACTIVE groups were also compared with the use of that test. To compare the combined effect of animals' activity and spine content on the selected parameters (the length of single-synapse spine and its neck, the diameter of doublesynapse spine head and neck, PSD area of excitatory and inhibitory synapses of double synapse-spines, the number of polyribosomes in double-synapse spines), 2-way ANOVA with post hoc Bonferroni test preceded by Kolmogorov-Smirnov normality test was used. To facilitate the evaluation of statistically significant results, the Cohen effect size (Cohen $d$; Lakens, 2013) was calculated.

To test the relationships between the single- and doublesynapse spine parameters that change over the course of a day and under different light conditions, Pearson correlation coefficient was used.

In Results and in graphs, data are presented as means \pm SD and means \pm SEM, respectively.

\section{RESULTS}

\section{Volume of Dendritic Spines}

No significant circadian or diurnal changes were observed in the total volume [single: 2-way ANOVA, $F_{\text {phase }}(1,197)=0.66$, $P=0.417$; double: 2 -way ANOVA, $\left.F_{\text {phase }}(1,85)=2.62, P=0.109\right]$, as well as in the volume of spine heads [single: 2-way ANOVA, $F_{\text {phase }}(1,197)=0.74, P=0.391$; double: 2 -way ANOVA, $F_{\text {phase }}(1,85)=1.52, P=0.220$ ] and necks [single: 2-way ANOVA, $F_{\text {phase }}(1,197)=0.25, P=0.616$; double: 2 -way ANOVA, $\left.F_{\text {phase }}(1,85)=2.01, P=0.160\right]$ in single- and double-synapse 


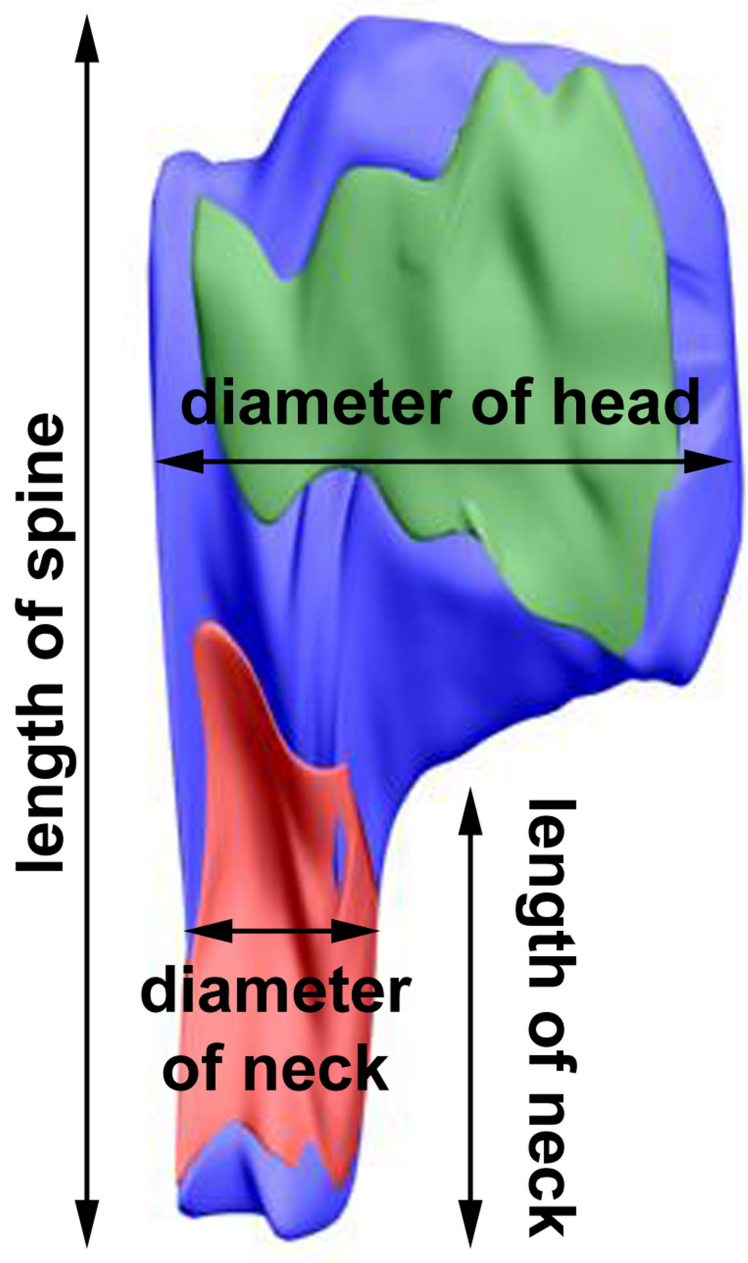

FIGURE 2 | 3D reconstruction of a representative double-synapse spine showing the measured parameters. Red area, inhibitory synapse; green area, excitatory synapse.

spines under LD and DD conditions. Similarly, there were no differences in the total volume [single: 2-way ANOVA, $F_{\text {condition }}(1,197)=1.36, P=0.245$; double: 2 -way ANOVA, $\left.F_{\text {condition }}(1,85)=0.21, P=0.644\right]$, the volume of spines heads [single: 2-way ANOVA, $F_{\text {condition }}(1,197)=2.55, P=0.112$; double: 2-way ANOVA, $\left.F_{\text {condition }}(1,85)=0.05, P=0.827\right]$ and necks [single: 2-way ANOVA, $F_{\text {condition }}(1,197)=0.003$, $P=0.956$; double: 2 -way ANOVA, $F_{\text {condition }}(1,85)=0.40$, $P=0.531]$ in single- and double-synapse spines between LD and DD conditions.

\section{Total Length of Dendritic Spines and Length of Spine Necks}

In the LD group, single-synapse spines were moderately longer (Cohen $d=0.58$ ) in the subgroup REST compared to ACTIVE [2-way ANOVA, $P<0.05, t=2.263 ; F_{\text {phase }}(1,197)=3.87$, $P=0.051$; Figure 3A $]$. In addition, we found similar differences in the length of single-synapse spine necks between the subgroups
REST and ACTIVE [medium effect size, Cohen $d=0.68$; 2 -way ANOVA, $P<0.05, t=2.663 ; F_{\text {phase }}(1,197)=1.89$, $P=0.171$; Figure $3 \mathrm{C}]$.

On the contrary, no significant differences in the length of single-synapse spines and spine necks between the subgroups REST and ACTIVE were observed in the DD group (Figures 3A,C).

In both groups (LD and DD), no significant differences between the phases were observed in the length of doublesynapse spines or in the length of double-synapse spine necks [REST/ACTIVE; 2-way ANOVA, spine length: $F_{\text {phase }}(1,85)=1.90 ; P=0.172$, spine neck length: $F_{\text {phase }}(1,85)=$ $3.35, P=0.071$; Figures 3B,D].

There were also no differences in the parameters between LD and DD groups in both single-synapse [2-way ANOVA, spine length: $F_{\text {condition }}(1,197)=0.05, P=0.827$, spine neck length: $\left.F_{\text {condition }}(1,197)=0.009, P=0.923\right]$ and double-synapse spines [2-way ANOVA, spine length: $F_{\text {condition }}(1,85)=0.008$, $P=0.927$; spine neck length: $F_{\text {condition }}(1,85)=0.07, P=0.787$; Figures 3A-D].

\section{Diameter of Spine Heads and Necks}

In both LD and DD groups, the diameters of single-synapse spine heads did not differ between the subgroups REST and ACTIVE [2-way ANOVA, $F_{\text {phase }}(1,197)=0.50, P=0.480$; Figure 4A], whereas the diameters of double-synapse spine heads were moderately (Cohen $d=0.56$ ) and slightly (Cohen $d=0.41$ ) larger in the ACTIVE phase compared to REST in the LD and DD groups, respectively [2-way ANOVA, $F_{\text {phase }}(1,85)=4.61$, $P=0.034$; Figure 4B].

Significant differences were found in the head diameters of single- [2-way ANOVA, $F_{\text {condition }}(1,197)=6.24, P=0.013$ ] and double-synapse spines between LD and DD conditions [2-way ANOVA, $F_{\text {condition }}(1,85)=4.53, P=0.036$; Figures 4A,B].

The neck diameter of single-synapse spines did not change between the subgroups REST and ACTIVE irrespectively of the light condition [2-way ANOVA, $F_{\text {phase }}(1,197)=2.763, P=0.098$; Figure 5C], whereas the neck diameter of double-synapse spines was moderately larger (Cohen $d=0.76$ ) in the subgroup REST than in the subgroup ACTIVE only in LD conditions [2way ANOVA, $P<0.05, t=2.336 ; F_{\text {interaction }}(1,85)=9.04$, $P=0.0035$; Figure 4D].

There were no differences in the diameters of spine necks between LD and DD groups in both single- [2-way ANOVA, $F_{\text {condition }}(1,197)=0.33, P=0.568$; Figure $\left.4 \mathrm{C}\right]$ and double-synapse spines [2-way $\operatorname{ANOVA,~} F_{\text {condition }}(1,85)=0.76$, $P=0.387$; Figure 4D].

\section{Area of PSD}

There were no differences in the PSD area of excitatory synapses in single-synapse spines between the subgroups REST and ACTIVE in both LD and DD groups [2-way ANOVA, $\left.F_{\text {phase }}(1,197)=3.41, P=0.066\right]$, as well as between LD and DD conditions [2-way ANOVA, $F_{\text {condition }}(1,197)=0.09$, $P=0.759$; Figure 5A].

The PSD areas of excitatory synapses localized on double-synapse spines were slightly (Cohen $d=0.40$ ) 

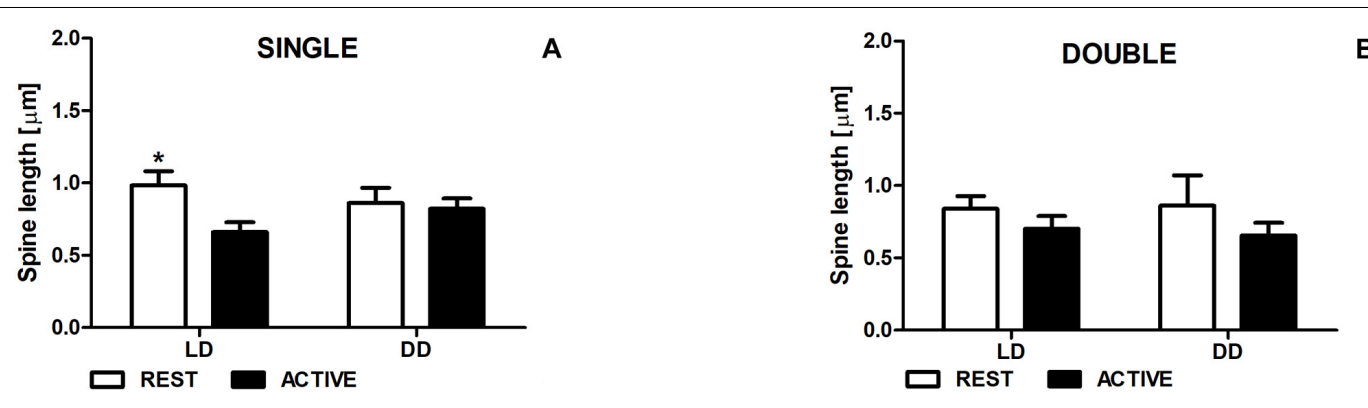

C

D
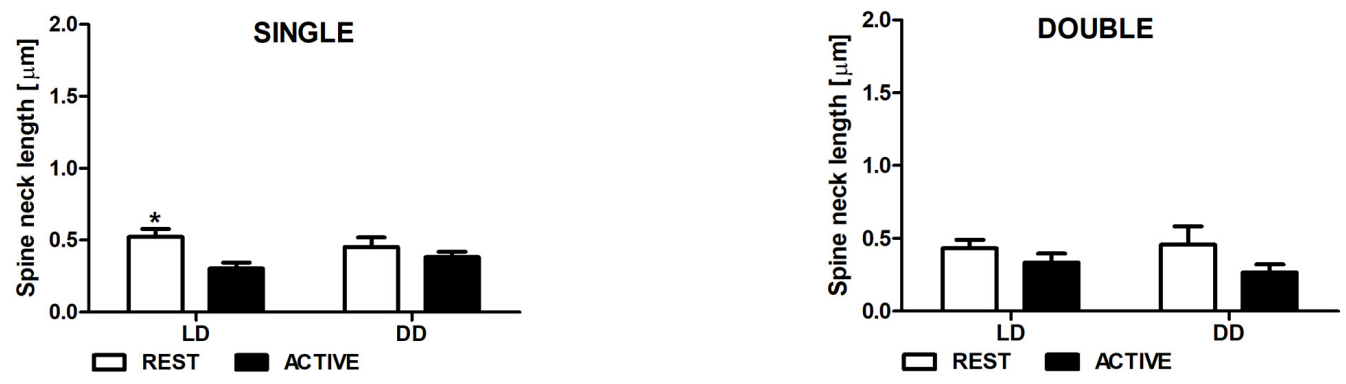

FIGURE $\mathbf{3}$ | Changes of the length of single-synapse spines and their necks are driven by the light. Spine length $(\mathbf{A}, \mathbf{B})$ and spine neck length $(\mathbf{C}, \mathbf{D})$ in REST and ACTIVE groups under LD and DD conditions. All graphs show means \pm SEM (2-way ANOVA, $\left.{ }^{\star} P<0.05\right)$. $N=4$ mice per group.

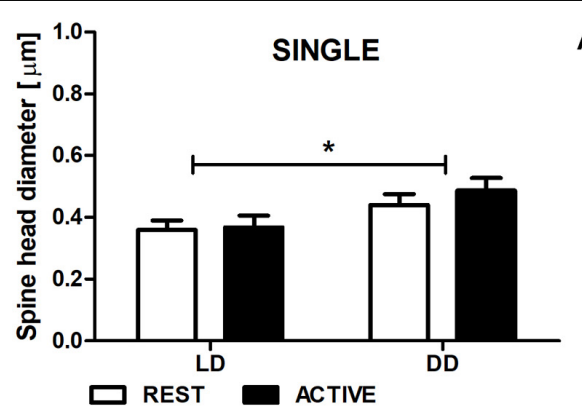

C

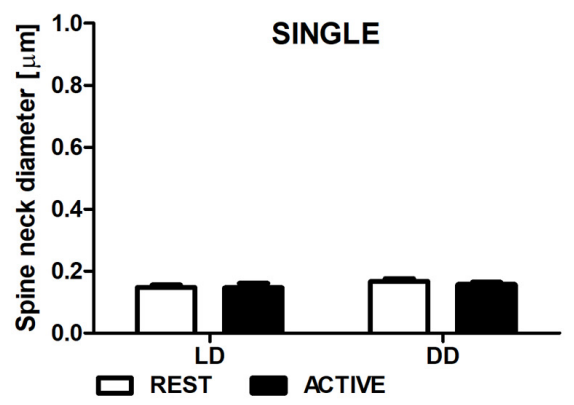

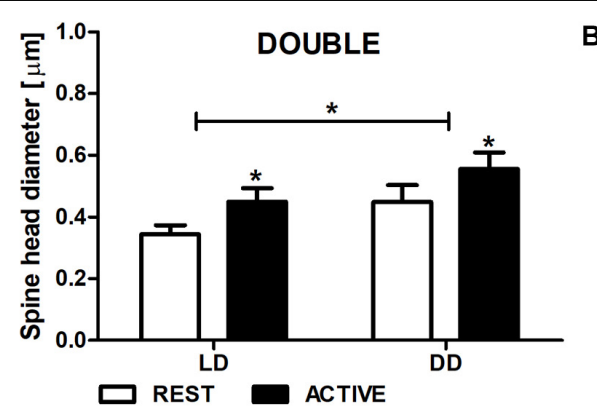

D

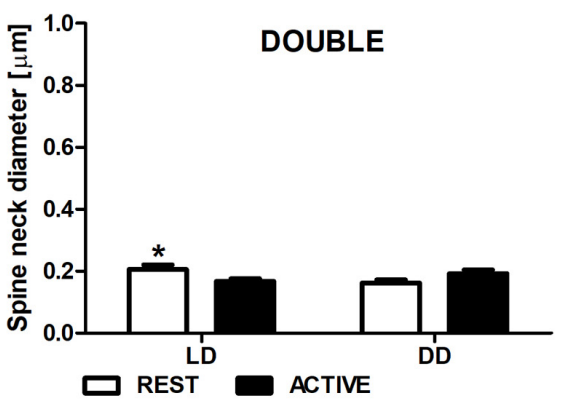

FIGURE 4 | Neck diameter changes in double-synapse spines are driven by the light. Diameter of spine heads (A,B) and necks (C,D) in groups REST and ACTIVE under LD and DD conditions. All graphs show means \pm SEM (2-way ANOVA, $\left.{ }^{*} P<0.05\right) . N=4$ mice per group. 

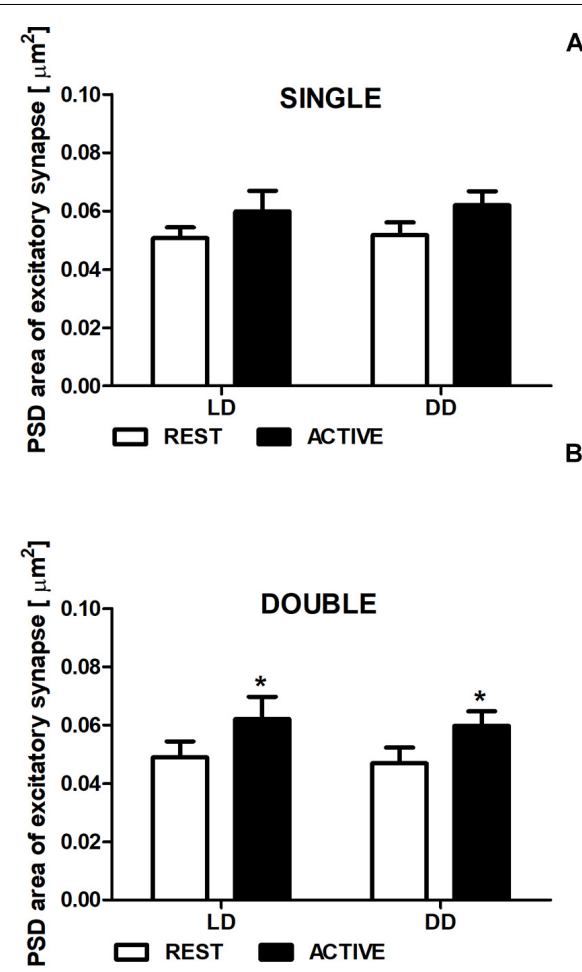

A

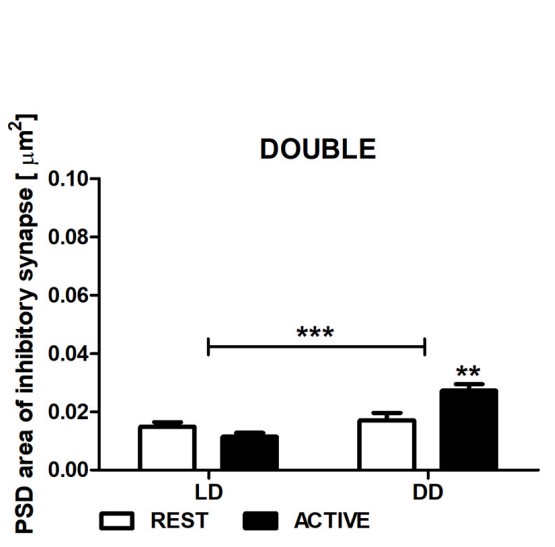

FIGURE 5 | Changes of PSD area of inhibitory synapses in double-synapse spines are driven by both circadian clock/locomotor activity and light. PSD area of excitatory (A,B) and inhibitory synapses $\mathbf{( C )}$ in groups REST and ACTIVE under LD and DD conditions. All graphs show means \pm SEM (2-way ANOVA, ${ }^{\star} P<0.05$, $\left.{ }^{\star \star} P<0.01,{ }^{\star \star \star} P<0.001\right) . N=4$ mice per group.

and moderately (Cohen $d=0.51$ ) larger in the ACTIVE phase compared to REST in the LD and DD groups, respectively [2-way ANOVA, $F_{\text {phase }}(1,85)=4.33, P=0.040$; Figure 5B), whereas the PSD area of inhibitory synapses was substantially larger (Cohen $d=0.88$ ) in the subgroup ACTIVE compared to the subgroup REST in the DD group [2-way ANOVA, $P<0.01, t=3.557 ; F_{\text {interaction }}(1,85)=10.88$, $P=0.0014$; Figure 5C].

Moreover, significant differences in the PSD area of inhibitory synapses were found between $\mathrm{LD}$ and $\mathrm{DD}$ conditions [2-way ANOVA, $F_{\text {condition }}(1,85)=19.38, P<0.0001$; Figure 5C], whereas in the PSD area of excitatory synapses localized on doublesynapse spines did not differ between LD and DD groups [2-way ANOVA, $F_{\text {condition }}(1,85)=0.12, P=0.725$; Figure 5B] .

The exemplary differences in single-synapse spine length, neck length and head diameter, double-synapse spine head and neck diameter, and PSD area of excitatory and inhibitory synapses are presented in Figure 6.

\section{Number and Distribution of Polyribosomes in Dendritic Spines}

In the LD group, no significant differences in the number of polyribosomes in single- [2-way ANOVA, $F_{\text {phase }}(1,197)=0.97$, $P=0.326$ ] and double-synapse spines [2-way ANOVA, $F_{\text {phase }}(1,85)=1.89, P=0.173$ ] were found between the subgroups REST and ACTIVE (Figures 7A,B).
In the DD group, the number of polyribosomes in doublesynapse spines was moderately higher (Cohen $d=0.67$ ) in the subgroup ACTIVE than in the subgroup REST [2way ANOVA, $P<0.05, t=2.704 ; F_{\text {interaction }}(1,85)=5.637$, $P=0.020$; Figure 7B], whereas there were no differences between the subgroups in single-synapse spines [REST/ACTIVE; 2-way ANOVA, $F_{\text {interaction }}(1,197)=2.76, P=0.098$; Figure 7A]

However, the differences in the number of polyribosomes were found in single- [2-way ANOVA, $F_{\text {condition }}(1,197)=6.44$, $P=0.012]$ and double-synapse spines [2-way ANOVA, $\left.F_{\text {condition }}(1,85)=15.31, P=0.0002\right]$ between $\mathrm{LD}$ and $\mathrm{DD}$ conditions (Figures 7A,B).

Irrespective of spine type and light conditions, the majority of spines contained more polyribosomes in the heads [single: $75.16 \% \pm 2.97 \%$, double: $76.17 \% \pm 4.06 \%$ ] than in the necks [single-2-way ANOVA, LD REST: $t=10.980, P<0.001$; LD ACTIVE: $t=7.915, P<0.001 ; F_{\text {location }}(1,12)=178.5, P<0.0001$; DD REST: $t=17.590, P<0.001$; DD ACTIVE: $t=17.490$, $P<0.001 ; F_{\text {location }}(1,12)=615.0, P<0.0001$; double-2way ANOVA, LD REST: $t=7.491, P<0.001$; LD ACTIVE: $t=10.250, P<0.001 ; F_{\text {location }}(1,12)=157.4, P<0.0001$; DD REST: $t=9.358, P<0.001$; DD ACTIVE: $t=7.292$, $\left.P<0.001 ; F_{\text {location }}(1,12)=138.6, P<0.0001\right]$. There were no differences in the distribution of polyribosomes between the subgroups REST and ACTIVE in both light conditions [singleLD: 2-way ANOVA, $F_{\text {phase }}(1,12)=0.00, P>0.99$; DD: 2 -way ANOVA, $F_{\text {phase }}(1,12)=0.00, P>0.99$; double-LD: 2 -way 

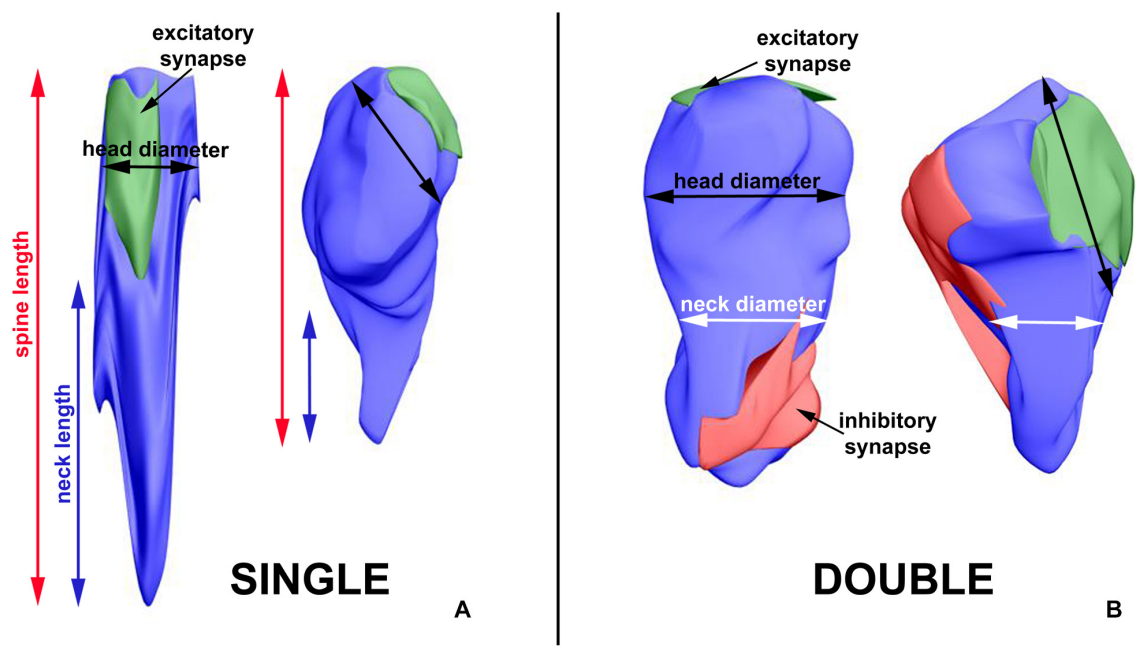

DOUBLE

FIGURE 6 | 3D reconstructions showing exemplary differences in single-synapse spine length, neck length and head diameter (A), double-synapse spine head and neck diameter and PSD area of excitatory and inhibitory synapses (B). The left sides of A and B-spines from LD REST group, the right sides - from DD ACTIVE group.
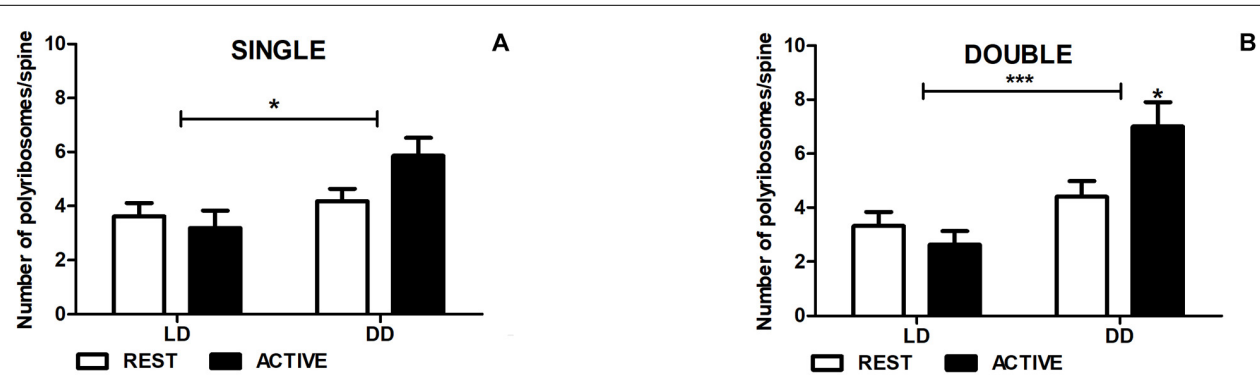

C
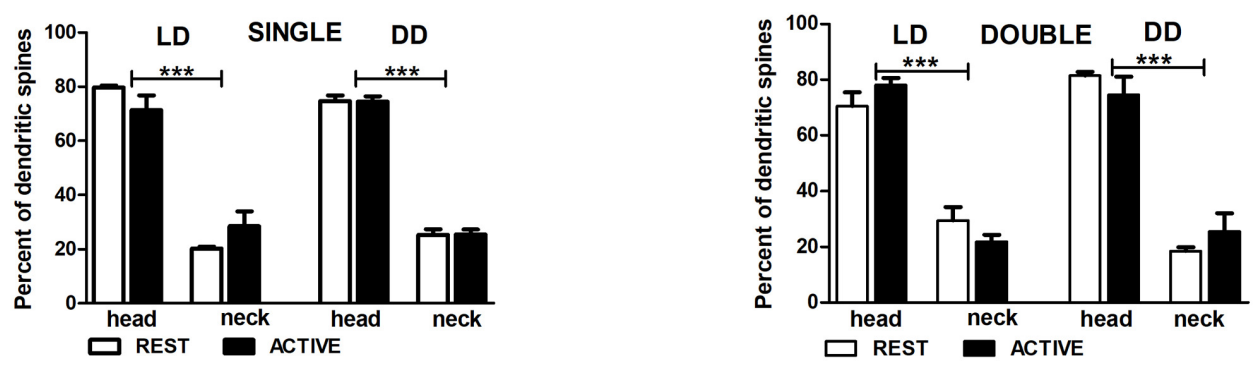

FIGURE 7 | Changes of polyribosome number in double-synapse spines are driven by both circadian clock/locomotor activity and light. Number of polyribosomes in spines $(\mathbf{A}, \mathbf{B})$ and their distribution $(\mathbf{C}, \mathbf{D})$ in groups REST and ACTIVE under LD and DD conditions. All graphs show means \pm SEM $(2-$ way ANOVA, * $P<0.05$, $\left.{ }^{* \star *} P<0.001\right) . N=4$ mice per group.

ANOVA, $F_{\text {phase }}(1,12)=0.00, P>0.99 ;$ DD: 2-way ANOVA, $F_{\text {phase }}(1,12)=0.00, P>0.99$; Figures 7C,D].

\section{Correlations of the Number of Polyribosomes and PSD Area of Synapses With Other Spine Parameters}

The number of polyribosomes was not correlated with the diameter of spine head in single- (Pearson correlation coefficient, $r=-0.04, P=0.600$; Figure 8A) and double-synapse spines (Pearson correlation coefficient, $r=-0.066, P=0.605$; Figure 8B). Similarly, no correlation was found between the diameter of double-synapse spine head and PSD area of excitatory synapses (Pearson correlation coefficient, $r=0.078$, $P=0.476$; Figure 8E); however, such (positive) correlation occurred in case of inhibitory synapses (Pearson correlation coefficient, $r=0.277, P=0.009$; Figure 8F). Moreover, the number of polyribosomes in double-synapse spines was 
positively correlated with PSD area of inhibitory synapses (Pearson correlation coefficient, $r=0.346, P=0.0009$; Figure 8D), but not of excitatory synapses (Pearson correlation coefficient, $r=0.204, P=0.056$; Figure 8C).

\section{Relationship Between Circadian Changes in Geometric Parameters and Spine Content}

To answer the question whether the content of the spines influences the cyclic changes of their geometric parameters, we selected for further analysis only the parameters that differed between the activity phases of the animals. Single- and double-synapse spines were divided into three subgroups: spines without any organelles (sER-free spines), spines containing sER, and spines containing SA (Jasinska et al., 2019).

In the LD group, total spine length was substantially longer in sER-free single-synapse spines (Cohen $d=0.99$ ) and in single-synapse spines containing SA (Cohen $d=0.81$ ) in the REST phase compared to ACTIVE [2-way ANOVA, $F_{\text {phase }}(1,73)=5.69, P=0.020$; Figure $\left.9 \mathrm{~A}\right]$. Similarly, the neck length of single-synapse spines was substantially longer in the spines with the same content (sER-free: Cohen $d=1.13$, SA: Cohen $d=1.12$ ) in the subgroup REST compared to ACTIVE [2-way ANOVA, $F_{\text {phase }}(1,73)=8.16, P=0.006$; Figure 9B]. Significant differences were also found in the neck diameters of sER-free double-synapse spines (large effect size, Cohen $d=1.51$ ) and double-synapse spines containing sER (large effect size, Cohen $d=0.94$ ) between the REST and ACTIVE subgroups [2-way ANOVA, $F_{\text {phase }}(1,37)=6.51$, $P=0.015$; Figure 9C]. On the other hand, no significant differences in the head diameter of double-synapse spines irrespective of spine content and light conditions were found between the subgroups REST and ACTIVE [2-way ANOVA, LD: $F_{\text {phase }}(1,37)=3.01, P=0.091 ; F_{\text {content }}(2,37)=0.12, P=0.889$; DD: $F_{\text {phase }}(1,41)=1.18, P=0.285 ; F_{\text {content }}(2,41)=2.86$, $P=0.068]$.

PSD areas of excitatory and inhibitory synapses did not differ irrespective of double-synapse spine content between activity phases in the LD group [2-way ANOVA, $F_{\text {phase }}(1,37)=1.76$, $P=0.192 ; F_{\text {content }}(2,37)=1.09, P=0.344$; Figures 9E,F], whereas in the DD group, double-synapse spines containing SA had substantially larger (Cohen $d=1.57$ ) PSD area of excitatory synapses than sER-free double-synapse spines only in the subgroup ACTIVE [2-way ANOVA, $t=2.862$, $P<0.05 ; F_{\text {content }}(2,41)=5.64, P=0.007$; Figure 9E] . Additionally, double-synapse spines containing sER as well as containing SA had substantially larger PSD area of inhibitory synapses during ACTIVE phase compared to REST in the DD group [sER: Cohen $d=1.34, t=2.882$, $P<0.05$; SA: Cohen $d=1.49 t=2.866, P<0.05$; 2-way ANOVA, $F_{\text {phase }}(1,41)=10.4, P=0.003 ; F_{\text {content }}(2,37)=0.12$, $P=0.889$; Figure 9F].

In the LD group, no significant differences in the number of polyribosomes in double-synapse spines with different content were found between the subgroups REST and
ACTIVE [2-way ANOVA, $F_{\text {phase }}(1,37)=0.77, P=0.387$; $\left.F_{\text {content }}(2,37)=3.15, P=0.055\right]$, whereas in the $\mathrm{DD}$ group, the number of polyribosomes in double-synapse spines containing SA was moderately higher (Cohen $d=0.67$ ) in the subgroup ACTIVE compared to REST [2-way ANOVA, $\left.t=2.758, P<0.05 ; F_{\text {phase }}(1,41)=4.77, P=0.035\right]$, as well as compared with sER-free double-synapse spines in the subgroup ACTIVE [large effect size, Cohen $d=0.98$; 2 -way ANOVA, $t=2.417, P<0.05 ; F_{\text {interaction }}(2,41)=4.63$, $P=0.011 ;$ Figure 9D].

\section{DISCUSSION}

\section{Single-Synapse Spines}

It is known that the changes in synapse strength are reflected by various modifications of spine geometry affecting both, head and neck of the spine (Fifkova, 1985; Araya et al., 2014; Bosch et al., 2014; Nishiyama and Yasuda, 2015). Single-synapse spines showed no change in volume regardless of the phase of the day and light conditions (day/night under LD condition and subjective day/subjective night under DD condition). It was also consistent with the absence of circadian changes in PSD area of excitatory synapses.

On the other hand, the total length and length of the neck of single-synapse spines are driven by light. The length of spine neck can affect the strength of excitatory synapses located on spine heads (Araya et al., 2014). There are no correlations between head size, PSD size, and neck length (Benavides-Piccione et al., 2002; Arellano et al., 2007), indicating that these parameters are independently regulated, and their contributions to synapse strength are not interrelated.

The observed changes in the length of single-synapse spines and the length of their necks concern single-synapse spines without organelles and containing SA. Our previous study showed that single-synapse spines containing SA, regardless of their shape, were not affected by the light (Jasinska et al., 2019). The present results indicate that although light does not change the number of these spines, it could modify their morphology.

In the somatosensory cortex, an increase in the number of excitatory synapses on the spines was found only as the effect of light under LD conditions, whereas an increase in the number of single-synapse spines was observed both, in the light phase of LD regime and during the subjective day under DD conditions (Jasinska et al., 2014; Jasinska et al., 2015). This study shows that the light also promotes elongation of singlesynapse spine necks. Interestingly, under constant darkness the number of excitatory synapses on all spines does not change (Jasinska et al., 2015), and the necks of single-synapse spines do not elongate.

However, during the subjective day, there are more single-synapse spines, what probably results from doubleto single-synapse spines transformation and requires the removal of inhibitory synapses from double-synapse spines (Jasinska et al., 2015). The number of singlesynapse spines is controlled by the biological clock 


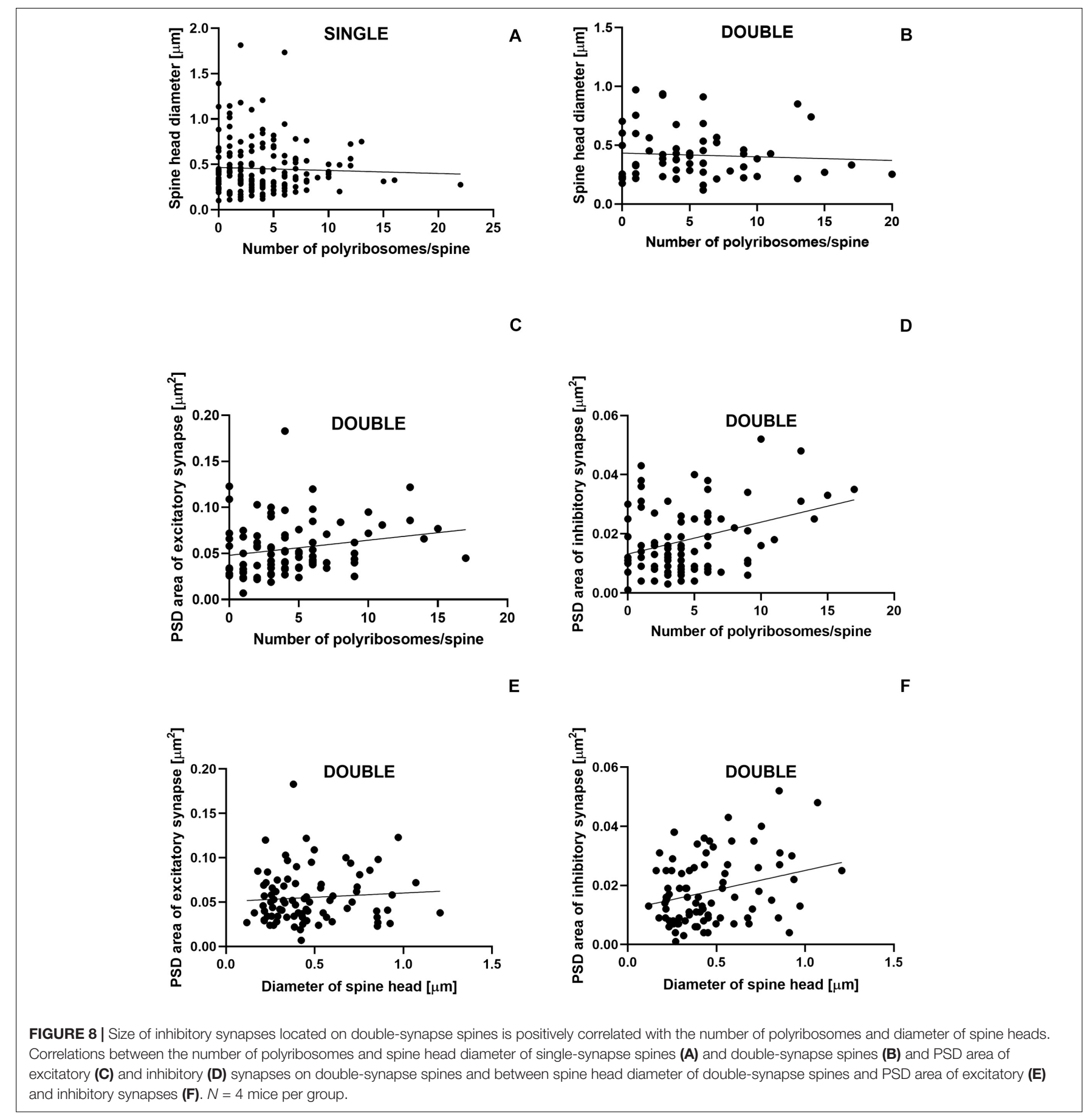

(Jasinska et al., 2015), whereas the efficiency of excitatory synapses on the spines seems to be regulated differently, depending on the light conditions: under LD conditions by changes in spine geometry and in constant darkness by cyclic formation and degradation of inhibitory synapses as well as transformation between single- and double-synapse spines.

It could be supposed that the changes in spine geometry are much faster than the process of formation and breakdown of inhibitory synapses; hence, the stress, such as the light for nocturnal animals, supports spine modification allowing more rapid execution of excitatory signaling.

Moreover, there were differences in the diameters of the single-synapse spine heads, which were larger in constant darkness compared to LD, although this effect was not accompanied by an increase in the head volume (suggesting the change of head shape) or in PSD area of excitatory synapses. Similar differences between the light conditions were observed in 

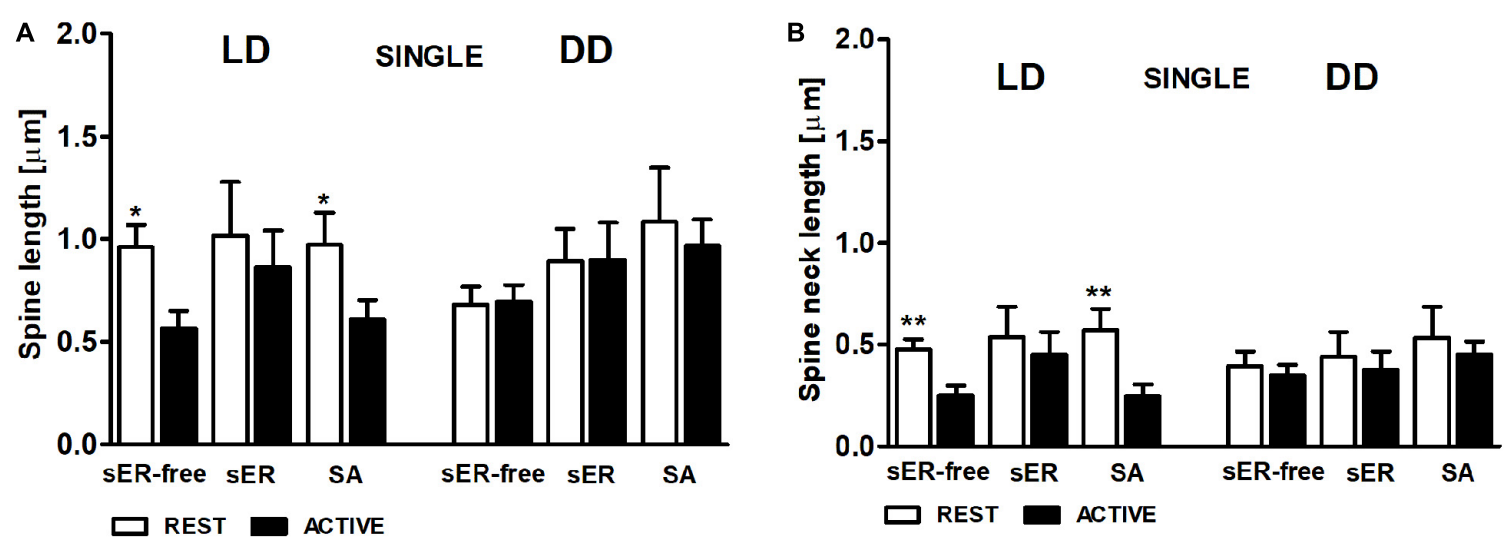

C

D
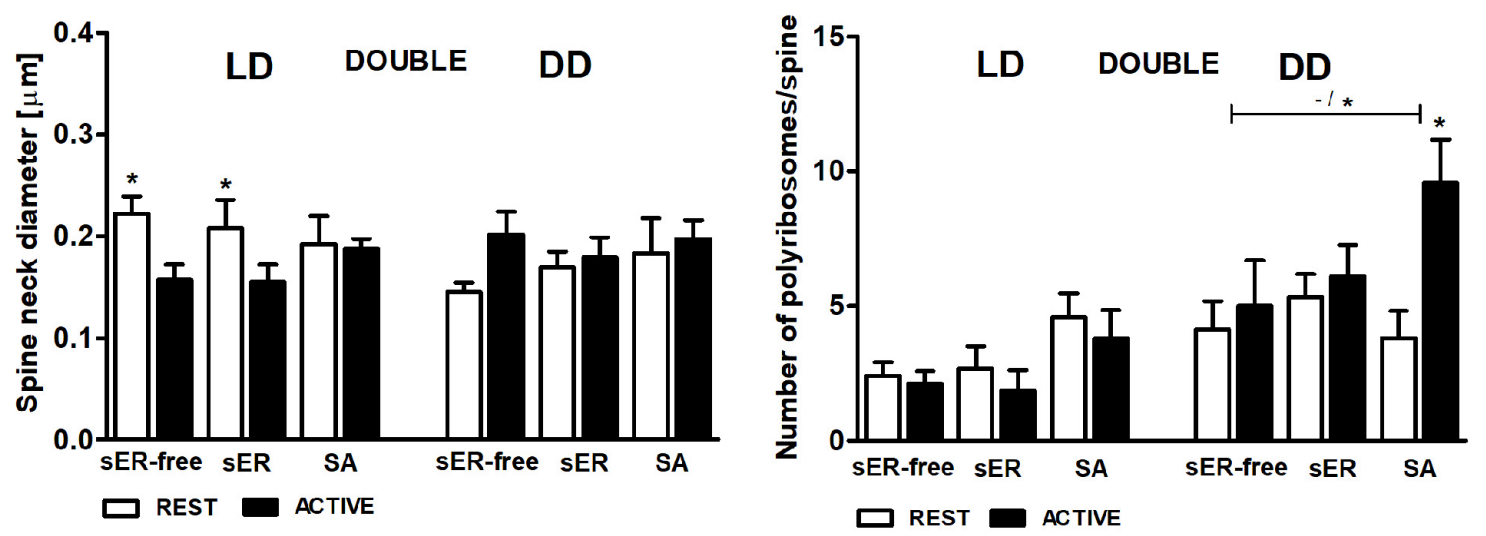

E

$\mathbf{F}$
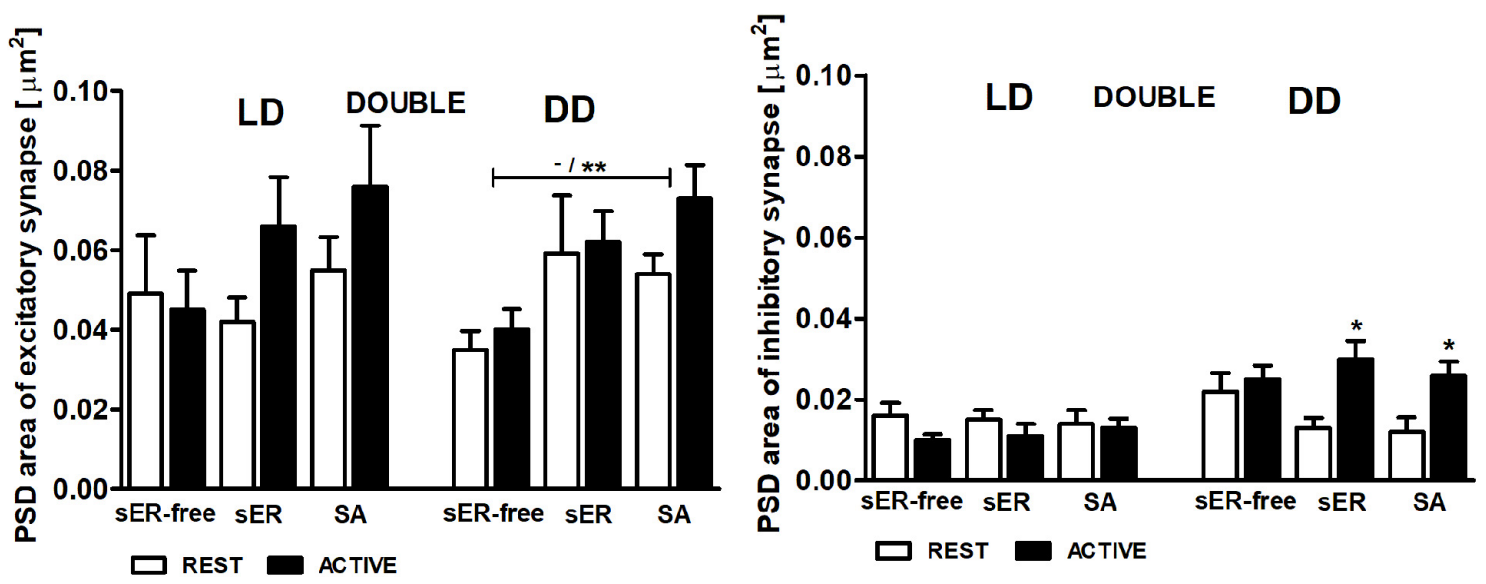

FIGURE 9 | Relationship between geometric parameters and spine content (spines sER-free, containing sER, and containing SA) during diurnal and circadian cycles. Spine length (A), spine neck length (B) of single-synapse spines, spine neck diameter (C), number of polyribosomes per spine (D), and PSD area of excitatory (E) and inhibitory (F) synapses of double-synapse spines in groups REST and ACTIVE under LD and DD conditions. All graphs show means \pm SEM (2-way ANOVA, ${ }^{*} P<0.05,{ }^{* *} P<0.01$ ). N $=4$ mice per group. The asterisks above the bars indicate significant differences in the percentage of spines with the same content between activity phases (REST vs. ACTIVE), whereas the asterisks above lines show significant differences between spines with different contents for the corresponding REST/ACTIVE phase. 
the number of polyribosomes in single-synapse spines, but these parameters were not correlated.

\section{Double-Synapse Spines}

In double-synapse spines, no changes were observed in the total volume of spines or in the volume of the spine heads and necks regardless of light condition. On the other hand, the diameter of the neck increased during the day. The studies related to activitydependent neural plasticity have shown that the increase in the diameter of spine neck is usually accompanied by its shortening (Fifkova and Anderson, 1981; Jasinska et al., 2006; Tønnesen et al., 2014). These morphological parameters of the neck are not correlated with each other (Arellano et al., 2007), but both reduce a resistance of the neck. Accordingly, in our study, although an increase in the neck diameter was observed, no changes were found in the length of the neck of double-synapse spines during the day irrespective of the light conditions. The day/night (under LD conditions) changes in the neck diameter were observed in double-synapse spines without sER/SA and containing sER, but not in double-synapse spines containing SA. This is in line with the fact that spines containing SA are considered to be the most stable spines (Knott et al., 2006; Bourne and Harris, 2008).

Although the modifications of the size of single-synapse spine neck could directly change (reduce or increase) the signal of the excitatory synapse, double-synapse spines also contain on the neck the inhibitory synapse, which might additionally regulate excitatory synaptic transmission. During the day, the decrease in the number of double-synapse spines (Jasinska et al., 2015) and a simultaneous increase in the size of their neck areas could be considered as a mechanism balancing the fewer number of spines by enhancing the efficiency of their synapses.

Another interesting result is an increase in the diameter of spine heads in DD compared to LD conditions, observed in both types of spines; additionally, in double-synapse spines, there were differences between the day/subjective day and night/subjective night irrespective of spine content. PSD enlargement is usually correlated with enlargement of the spine head (Knott et al., 2006; Arellano et al., 2007), and it seems that changes of the diameter of double-synapse spine heads should be related to the modifications of excitatory synapses, because they are located on the heads, and their PSD area is modified, depending on the activity phase of the animals. Unexpectedly, these parameters are not correlated, whereas a positive correlation was found between the head diameter and the PSD area of inhibitory synapses. This result is especially interesting, because inhibitory synapses are usually located on the necks of double-synapse spines (Jasinska et al., 2006). Moreover, enlargement of PSD area in inhibitory synapses was accompanied by increase in the number of polyribosomes, suggesting an increase in the strength of these synapses observed only during the subjective night. The size of inhibitory synapses changed only in double-synapse spines containing sER or SA. Moreover, in double-synapse spines containing SA, synapse enlargement was accompanied by increase in the number of polyribosomes. The SA, in cooperation with polyribosomes, is responsible for the enlargement of spine head and increased accumulation of glutamatergic receptors, leading to enhancement of excitatory synapses (Vlachos et al., 2009; Ostroff et al., 2010; Jedlicka and Deller, 2017). It seems that SA could play a similar role in double-synapse spines and their inhibitory synapses during the circadian cycle. Similarly, the presence of sER in spines contributes to synaptic enlargement (Chirillo et al., 2019).

Table 1 summarizes the influence of the light and endogenous effect on the number and morphology of dendritic spines in mouse barrel cortex.

\section{Functional Significance of Changes in Spine Geometry}

Results of this study show that single- and double-synapse spines are differently involved in diurnal and circadian changes. Both types of spines regulate synaptic transmission by modifications of the morphology of their necks; however, different geometrical parameters are changed. In single-synapse spines, the neck length modification was observed, whereas double-synapse spines changed the diameter of their necks. Both types of changes are associated with the light phase of the diurnal cycle and have similar effects: regulate the resistance of spine neck, influence the degree of isolation of the spine from its parent dendrite, and even control the effectiveness of excitatory synapse placed on the spine head (Arellano et al., 2007; Araya et al., 2014). However, the changes observed in single- and double-synapse spines have opposite results: elongation of the neck in single-synapse spine increases the isolation of spine head from dendrite, whereas widening of the neck in doublesynapse spine increases communication between spine and dendrite. On the other hand, spines with wider and shorter necks might be less susceptible to subsequent changes, because second messengers diffuse faster into the dendritic shafts due to increased capacity of the spine neck, whereas additional depolarization is stronger in spines better isolated from dendrites (with longer and more narrow necks; Tønnesen et al., 2014; Tønnesen and Nägerl, 2016).

Although the length and diameter of spine neck are not correlated with each other (Arellano et al., 2007), in case of experience-dependent changes these parameters have been observed as mutually coupled; i.e., widening of spine neck was accompanied by its shortening (Jasinska et al., 2006; Tønnesen et al., 2014). Such effect was not observed in this study. It could be concluded that modification of only one of neck parameters does not disturb function of neurons of the somatosensory cortex in the diurnal cycle.

The number of excitatory synapses located on single-synapse spines increases during the subjective day (Jasinska et al., 2015), but they do not change their size, while during the subjective night, the number of inhibitory synapses increases (Jasinska et al., 2015), and they are larger. Generally, no morphological changes of the excitatory synapses located on single-synapses spines were observed in the diurnal and circadian cycle. On the contrary, PSD area of inhibitory synapses increased during the subjective night, indicating enhancement of the strength 
TABLE 1 | Influence of light and the circadian clock/locomotor activity on single- and double-synapse spines in mouse barrel cortex.

\begin{tabular}{|c|c|c|}
\hline & Single-synapse spines & Double-synapse spines \\
\hline \multirow[t]{2}{*}{ Effect of light } & $\begin{array}{l}\text { Increase in: number of intermediate sER-free spines (Jasinska } \\
\text { et al., 2019), total length of spine (especially sER-free and } \\
\text { containing SA), length of spine neck (especially sER-free and } \\
\text { containing SA) }\end{array}$ & $\begin{array}{l}\text { Increase in: stubby spines containing sER, mushroom spines } \\
\text { containing SA, thin/mushroom spines (Jasinska et al., 2019), } \\
\text { diameter of spine neck (especially sER-free and containing sER) }\end{array}$ \\
\hline & $\begin{array}{l}\text { Decrease in: number of stubby sER-free spines (Jasinska } \\
\text { et al., 2019), diameter of spine head, number of } \\
\text { polyribosomes }\end{array}$ & $\begin{array}{l}\text { Decrease in: number of stubby sER-free spines, number of } \\
\text { mushroom spines (especially sER-free) (Jasinska et al., 2019), } \\
\text { diameter of spine head, PSD area of inhibitory synapse, number of } \\
\text { polyribosomes }\end{array}$ \\
\hline \multirow[t]{2}{*}{$\begin{array}{l}\text { Effect of circadian } \\
\text { clock/locomotor activity } \\
\text { (endogenous effect) }\end{array}$} & $\begin{array}{l}\text { Increase in: number of thin spines containing sER } \\
\text { (Jasinska et al., 2019) }\end{array}$ & $\begin{array}{l}\text { Increase in: total number of spines (Jasinska et al., 2015), number } \\
\text { of mushroom spines containing sER (Jasinska et al., 2019), } \\
\text { diameter of spine head, PSD area of excitatory synapse, PSD area } \\
\text { of inhibitory synapse (especially containing sER and SA) number of } \\
\text { polyribosomes (especially in spines containing SA) }\end{array}$ \\
\hline & $\begin{array}{l}\text { Decrease in: total number of spines (Jasinska et al., 2015), } \\
\text { number of stubby spines containing SA (Jasinska et al., 2019) }\end{array}$ & $\begin{array}{l}\text { Decrease in: number of thin spines containing sER, number of } \\
\text { mushroom sER-free spines (Jasinska et al., 2019) }\end{array}$ \\
\hline $\begin{array}{l}\text { Effect of both factors (light } \\
\text { and endogenous effect) }\end{array}$ & $\begin{array}{l}\text { Increase in: number of stubby spines containing sER, number } \\
\text { of mushroom spines (especially containing sER; } \\
\text { Jasinska et al., 2019) }\end{array}$ & $\begin{array}{l}\text { Increase in: number of thin spines (especially sER-free; } \\
\text { Jasinska et al., 2019) }\end{array}$ \\
\hline
\end{tabular}

of these synapses. Such enhancement might also have more complex consequence, because strong inhibitory synapses located on double-synapse spines could more efficiently control/regulate the effectiveness of excitatory synapses, affecting the conductivity of the whole spine.

The previous study showed that the number of mushroom double-synapse spines containing SA, i.e., the most mature and stable spines, increases in the light (Jasinska et al., 2019). Interestingly, in constant darkness, the increase in the size of inhibitory synapses correlated with the increase in the number of polyribosomes during the subjective night also concerns doublesynapse spines containing SA. This suggests that double-synapse spines with SA are regulated at various levels by both light and endogenous factor and play a special role in diurnal and circadian rhythms.

As appears from this and previous studies (Jasinska et al., $2015,2019)$, the function of single- and double-synapse spines in the somatosensory cortex is different during diurnal and during circadian cycle. It seems that in the circadian cycle (under DD conditions), regulation of synaptic transmission is largely based on the inhibitory synapses. Even the changes in the number of single-synapse spines seem to be the consequence of addition/subtraction of inhibitory synapses on double-synapse spines. In the diurnal cycle (under LD conditions), however, both single- and double-synapse spines are equally involved in the regulation of the received inputs, although they regulate them in different ways.

\section{Implications for Sleep Theories}

The synaptic homeostasis hypothesis (Tononi and Cirelli, 2006) presumes strengthening of the synaptic network during the activity phase of animals and weakening during the rest phase. It seems that an opposite trend occurs in the barrel cortex. By enhancing inhibitory transmission in the night/subjective night, overall synaptic transmission is weakened during higher activity of animals.
The results obtained in this study seem to confirm the homeostatic synaptic plasticity hypothesis (Turrigiano and Nelson, 2000; Pozo and Goda, 2010), which assumes the existence of an adaptive compensatory mechanism. In the night/subjective night, the number of inhibitory synapses on the spines increases (Jasinska et al., 2015), and although only during constant darkness, the inhibitory synapses are larger, what is associated with an increase in their strength and consequently leads to weakening of synaptic transmission, being a compensation for excitation resulting from increased activity of animals.

The elongation of single-synapse spines and their necks observed during the day (light phase) under LD conditions also leads to reduced excitatory transmission from spine to the parent dendrite. Interestingly, the number of excitatory synapses increases in the light phase of the diurnal cycle (Jasinska et al., 2015), but due to changes in synapse morphology they are less effective. It seems that the presence of more excitatory synapses during that phase suggests preparation for more stressful conditions but not necessarily does mean a direct increase in excitation. Additionally, the widening of the necks in double-synapse spines has similar significance, because it leads to easier control of excitatory transmission by the inhibitory synapses also present on these spines.

\section{Study Strengths, Limitations, and Future Research Directions}

This study shows for the first time the circadian changes of measurable spine geometric parameters using a rigorous quantitative approach offered by transmission electron microscopy. However, it has certain limitations. We have not performed an a priori power analysis, and although the number of spines in the groups is large, we cannot be sure whether it is sufficient to show all existing differences between the groups. Nevertheless, we selected to the study all spines that were completely contained within the sample volume and could serve for $3 \mathrm{D}$ reconstruction. 
Another limitation of our study is the selection of just two time points across the 24-h cycle. Although they correspond well to changes in animal activity (REST/ACTIVE), we cannot separate the role of the biological clock from the direct influence of the locomotor activity of animals. Further studies using additional time points or another approach (e.g., clock mutants) are necessary to elucidate that question.

This study was conducted on dendritic spines obtained exclusively from the barrel field of the somatosensory cortex. Because significant differences in the cyclic changes of dendritic spine density were found depending on regions of the brain and even between the hemispheres, our results cannot be generalized to the entire neocortex. It seems appropriate to investigate dendritic spines in other regions of the cortex, e.g., in the visual cortex, where light plays a different role. Such comparison of cyclic changes in the geometric parameters of spines between different areas of the brain would significantly increase our understanding of the diurnal and circadian rhythms.

\section{DATA AVAILABILITY STATEMENT}

All datasets presented in this study are included in the article.

\section{REFERENCES}

Acosta-Peña, E., Camacho-Abrego, I., Melgarejo-Gutiérrez, M., Flores, G., Drucker-Colín, R., and García-García, F. (2015). Sleep deprivation induces differential morphological changes in the hippocampus and prefrontal cortex in young and old rats. Synapse 69, 15-25. doi: 10.1002/syn.21779

Araya, R., Jiang, J., Eisenthal, K. B., and Yuste, R. (2006). The spine neck filters membrane potentials. Proc. Natl. Acad. Sci. U.S.A. 103, 17961-17966. doi: 10. 1073/pnas.0608755103

Araya, R., Vogels, T. P., and Yuste, R. (2014). Activity-dependent dendritic spine neck changes are correlated with synaptic strength. Proc. Natl. Acad. Sci. U.S.A. 111, E2895-E2904. doi: 10.1073/pnas.1321869111

Areal, C. C., Warby, S. C., and Mongrain, V. (2017). Sleep loss and structural plasticity. Curr. Opin. Neurobiol. 44, 1-7. doi: 10.1016/j.conb.2016. 12.010

Arellano, J. I., Benavides-Piccione, R., DeFelipe, J., and Yuste, R. (2007). Ultrastructure of dendritic spines: correlation between synaptic and spine morphologies. Front. Neurosci. 1, 131-143. doi: 10.3389/neuro.01.1.1.010.2007

Balkema, G. W., Cusick, K., and Nguyen, T. H. (2001). Diurnal variation in synaptic ribbon length and visual threshold. Vis. Neurosci. 18, 789-797. doi: 10.1017/ s0952523801185123

Becquet, D., Girardet, C., Guillaumond, F., François-Bellan, A. M., and Bosler, O. (2008). Ultrastructural plasticity in the rat suprachiasmatic nucleus. Possible involvement in clock entrainment. Glia 56, 294-305. doi: 10.1002/glia.20613

Behrens, U. D., Kasten, P., and Wagner, H. J. (1998). Adaptation-dependent plasticity of rod bipolar cell axon terminal morphology in the rat retina. Cell Tissue Res. 294, 243-251. doi: 10.1007/s004410051174

Benavides-Piccione, R., Ballesteros-Yáñez, I., DeFelipe, J., and Yuste, R. (2002). Cortical area and species differences in dendritic spine morphology. J. Neurocytol. 31, 337-346. doi: 10.1023/a:1024134312173

Bloodgood, B. L., and Sabatini, B. L. (2005). Neuronal activity regulates diffusion across the neck of dendritic spines. Science 310, 866-869. doi: 10.1126/science. 1114816

Bosch, M., Castro, J., Saneyoshi, T., Matsuno, H., Sur, M., and Hayashi, Y. (2014). Structural and molecular remodeling of dendritic spine substructures during long-term potentiation. Neuron 82, 444-459. doi: 10.1016/j.neuron.2014. 03.021

\section{ETHICS STATEMENT}

The animal study was reviewed and approved by Animal Care and Use Committees of the Jagiellonian University.

\section{AUTHOR CONTRIBUTIONS}

MJ and EP contributed to conception and design of the study. MJ and OW performed the experiments. MJ and EJ-G analyzed the data. MJ performed the statistical analysis. JL and GL participated in data interpretation. MJ wrote the draft of the manuscript. EP and JL revised the manuscript critically for important intellectual content. All authors contributed to the article and approved the submitted version.

\section{FUNDING}

This study was supported by the statutory grant (N41/DBS/000064) from the Jagiellonian University Medical College to MJ. The funders were not involved in study design, data collection and analysis, decision to publish, or preparation of the manuscript.

Bosch, M., and Hayashi, Y. (2012). Structural plasticity of dendritic spine. Curr. Opin. Neurobiol. 22, 383-388. doi: 10.1016/j.conb.2011.09.002

Bourne, J., and Harris, K. M. (2007). Do thin spines learn to be mushroom spines that remember? Curr. Opin. Neurobiol. 17, 381-386. doi: 10.1016/j.conb.2007. 04.009

Bourne, J. N., and Harris, K. M. (2008). Balancing structure and funcion at hippocampal dendritic spines. Annu. Rev. Neurosci. 31, 47-67. doi: 10.1146/ annurev.neuro.31.060407.125646

Bourne, J. N., and Harris, K. M. (2011). Coordination of size and number of excitatory and inhibitory synapses results in a balanced structural plasticity along mature hippocampal CA1 dendrites during LTP. Hippocampus 21, 354373. doi: 10.1002/hipo.20768

Cheifetz, P. (1971). The daily rhythm of the secretion of corticotrophin and corticosterone in rats and mice. J. Endocrinol. 49, xi-xii.

Chirillo, M. A., Waters, M. S., Lindsey, L. F., Bourne, J. N., and Harris, K. M. (2019). Local resources of polyribosomes and SER promote synapse enlargement and spine clustering after long-term potentiation in adult rat hippocampus. Sci. Rep. 9:3861. doi: 10.1038/s41598-019-40520-x

Chung, S., Son, G. H., and Kim, K. (2011). Circadian rhythm of adrenal glucocorticoid: its regulation and clinical implications. Biochim. Biophys. Acta 1812, 581-591. doi: 10.1016/j.bbadis.2011.02.003

Denk, W., Sugimori, M., and Llinás, R. (1995). Two types of calcium response limited to single spines in cerebellar Purkinje cells. Proc. Natl. Acad. Sci. U.S.A. 92, 8279-8282. doi: 10.1073/pnas.92.18.8279

Fernández, M. P., Berni, J., and Ceriani, M. F. (2008). Circadian remodeling of neuronal circuits involved in rhythmic behavior. PLoS Biol. 6:e69. doi: 10.1371/ journal.pbio.0060069

Fifkova, E. (1985). A possible mechanism of morphometric changes in dendritic spines induced by stimulation. Cell. Mol. Neurobiol. 5, 47-63. doi: 10.1007/ BF00711085

Fifkova, E., and Anderson, C. L. (1981). Stimulation-induced changes in dimensions of stalks of dendritic spines in the dentate molecular layer. Exp. Neurol. 74, 621-627. doi: 10.1016/0014-4886(81)90197-7

Frank, M. G. (2016). Circadian regulation of synaptic plasticity. Biology 5:31. doi: 10.3390/biology5030031

Frank, M. G., and Cantera, R. (2014). Sleep, clocks, and synaptic plasticity. Trends Neurosci. 37, 491-501. doi: 10.1016/j.tins.2014.06.005 
Geinisman, Y., Berry, R. W., Disterhoft, J. F., Power, J. M., and Van der Zee, E. A. (2001). Associative learning elicits the formation of multiple-synapse boutons. J. Neurosci. 21, 5568-5573. doi: 10.1523/JNEUROSCI.21-15-05568.2001

Geinisman, Y., Disterhoft, J. F., Gundersen, H. J. G., McEchron, M. D., Persina, I. S., Power, J. M., et al. (2000). Remodeling of hippocampal synapses after hippocampus-dependent associative learning. J. Comp. Neurol. 417, 49-59. doi: 10.1002/(SICI)1096-9861(20000131)417:1<49::AID-CNE4>3.0.CO;2-3

Girardet, C., Blanchard, M. P., Ferracci, G., Lévêque, C., Moreno, M., FrançoisBellan, A. M., et al. (2010). Daily changes in synaptic innervation of VIP neurons in the rat suprachiasmatic nucleus: contribution of glutamatergic afferents. Eur. J. Neurosci. 31, 359-370. doi: 10.1111/j.1460-9568.2009.07071.x

Górska-Andrzejak, J., Keller, A., Raabe, T., Kilianek, L., and Pyza, E. (2005). Structural daily rhythms in GFP-labelled neurons in the visual system of Drosophila melanogaster. Photochem. Photobiol. Sci. 4, 721-726. doi: 10.1039/ b417023g

Górska-Andrzejak, J., Makuch, R., Stefan, J., Görlich, A., Semik, D., and Pyza, E. (2013). Circadian expression of the presynaptic active zone protein bruchpilot in the lamina of Drosophila melanogaster. Dev. Neurobiol. 73, 14-26. doi: 10. 1002/dneu.22032

Havekes, R., Park, A. J., Tudor, J. C., Luczak, V. G., Hansen, R. T., Ferri, S. L., et al. (2016). Sleep deprivation causes memory deficits by negatively impacting neuronal connectivity in hippocampal area CA1. eLife 5:e13424. doi: 10.7554/ eLife. 13424

Ikeda, M., Hojo, Y., Komatsuzaki, Y., Okamoto, M., Kato, A., Takeda, T., et al. (2015). Hippocampal spine changes across the sleep-wake cycle: corticosterone and kinases. J. Endocrinol. 226, M13-M27. doi: 10.1530/JOE-15-0078

Ikeno, T., Weil, Z. M., and Nelson, R. J. (2013). Photoperiod affects the diurnal rhythm of hippocampal neuronal morphology of Siberian hamsters. Chronobiol. Int. 30, 1089-1100. doi: 10.3109/07420528.2013.800090

Jasinska, M., Grzegorczyk, A., Jasek, E., Litwin, J. A., Kossut, M., BarbackaSurowiak, G., et al. (2014). Daily rhythm of synapse turnover in mouse somatosensory cortex. Acta Neurobiol. Exp. 74, 104-110.

Jasinska, M., Grzegorczyk, A., Woznicka, O., Jasek, E., Kossut, M., BarbackaSurowiak, G., et al. (2015). Circadian rhythmicity of synapses in mouse somatosensory cortex. Eur. J. Neurosci. 42, 2585-2594. doi: 10.1111/ejn.13045

Jasinska, M., Jasek-Gajda, E., Woznicka, O., Lis, G. J., Pyza, E., and Litwin, J. A. (2019). Circadian clock regulates the shape and content of dendritic spines in mouse barrel cortex. PLoS One 14:e0225394. doi: 10.1371/journal.pone. 0225394

Jasinska, M., Siucinska, E., Cybulska-Klosowicz, A., Pyza, E., Furness, D. N., Kossut, M., et al. (2010). Rapid, learning-induced inhibitory synaptogenesis in murine barrel field. J. Neurosci. 30, 1176-1184. doi: 10.1523/JNEUROSCI.297009.2010

Jasinska, M., Siucinska, E., Głazewski, S., Pyza, E., and Kossut, M. (2006). Characterization and plasticity of the double synapse spines in the barrel cortex of the mouse. Acta Neurobiol. Exp. 66, 99-104.

Jasinska, M., Siucinska, E., Jasek, E., Litwin, J. A., Pyza, E., and Kossut, M. (2013). Fear learning increases the number of polyribosomes associated with excitatory and inhibitory synapses in the barrel cortex. PLoS One 8:e54301. doi: 10.1371/ journal.pone.0054301

Jasinska, M., Siucinska, E., Jasek, E., Litwin, J. A., Pyza, E., and Kossut, M. (2016). Effect of associative learning on memory spine formation in mouse barrel cortex. Neural Plast. 2016:9828517. doi: 10.1155/2016/9828517

Jedlicka, P., and Deller, T. (2017). Understanding the role of synaptopodin and the spine apparatus in Hebbian synaptic plasticity - New perspectives and the need for computational modeling. Neurobiol. Learn. Mem. 138, 21-30. doi: $10.1016 /$ j.nlm.2016.07.023

Kasai, H., Fukuda, M., Watanabe, S., Hayashi-Takagi, A., and Noguchi, J. (2010). Structural dynamics of dendritic spines in memory and cognition. Trends Neurosci. 33, 121-129. doi: 10.1016/j.tins.2010.01.001

Knott, G. W., Holtmaat, A., Wilbrecht, L., Welker, E., and Svoboda, K. (2006). Spine growth precedes synapse formation in the adult neocortex in vivo. Nat. Neurosci. 9, 1117-1124. doi: 10.1038/nn1747

Knott, G. W., Quairiaux, C., Genoud, C., and Welker, E. (2002). Formation of dendritic spines with GABAergic synapses induced by whisker stimulation in adult mice. Neuron 34, 265-273. doi: 10.1016/S0896-6273(02) 00663-3
Korkotian, E., and Segal, M. (2007). Morphological constraints on calcium dependent glutamate receptor trafficking into individual dendritic spine. Cell Calcium 42, 41-57. doi: 10.1016/j.ceca.2006.11.006

Kovalchuk, Y., Eilers, J., Lisman, J., and Konnerth, A. (2000). NMDA receptormediated subthreshold $\mathrm{Ca} 2+$ signals in spines of hippocampal neurons. J. Neurosci. 20, 1791-1799. doi: 10.1523/JNEUROSCI.20-05-01791.2000

Krzeptowski, W., Hess, G., and Pyza, E. (2018). Circadian plasticity in the brain of insects and rodents. Front. Neural Circuits 12:32. doi: 10.3389/fncir.2018.00032

Lakens, D. (2013). Calculating and reporting effect sizes to facilitate cumulative science: a practical primer for t-tests and ANOVAs. Front. Psychol. 4:863. doi: 10.3389/fpsyg.2013.00863

Leuner, B., and Shors, T. J. (2004). New spines, new memories. Mol. Neurobiol. 29, 117-130. doi: 10.1385/MN:29:2:117

Liston, C., Cichon, J. M., Jeanneteau, F., Jia, Z., Chao, M. V., and Gan, W. B. (2013). Circadian glucocorticoid oscillations promote learning- dependent synapse formation and maintenance. Nat. Neurosci. 16, 698-705. doi: 10.1038/nn.3387

Liston, C., and Gan, W. B. (2011). Glucocorticoids are critical regulators of dendritic spine development and plasticity in vivo. Proc. Natl. Acad. Sci. U.S.A. 108, 16074-16079. doi: 10.1073/pnas.1110444108

Maiti, P., Manna, J., Ilavazhagan, G., Rossignol, J., and Dunbar, G. L. (2015). Molecular regulation of dendritic spine dynamics and their potential impact on synaptic plasticity and neurological diseases. Neurosci. Biobehav. Rev. 59, 208-237. doi: 10.1016/j.neubiorev.2015.09.020

Maret, S., Faraguna, U., Nelson, A. B., Cirelli, C., and Tononi, G. (2011). Sleep and wake modulate spine turnover in the adolescent mouse cortex. Nat. Neurosci. 14, 1418-1420. doi: 10.1038/nn.2934

Mehnert, K. I., Beramendi, A., Elghazali, F., Negro, P., Kyriacou, C. P., and Cantera, R. (2007). Circadian changes in Drosophila motor terminals. Dev. Neurobiol. 67, 415-421. doi: 10.1002/dneu.20332

Nikonenko, I., Jourdain, P., Alberi, S., Toni, N., and Muller, D. (2002). Activityinduced changes of spine morphology. Hippocampus 12, 585-591. doi: 10.1002/ hipo. 10095

Nishiyama, J., and Yasuda, R. (2015). Biochemical computation for spine structural plasticity. Neuron 87, 63-75. doi: 10.1016/j.neuron.2015.05.043

Noguchi, J., Matsuzaki, M., Ellis-Davies, G. C. R., and Kasai, H. (2005). Spine-neck geometry determines NMDA receptor-dependent $\mathrm{Ca} 2+$ signaling in dendrites. Neuron 46, 609-622. doi: 10.1016/j.neuron.2005.03.015

Ostroff, L. E., Cain, C. K., Bedont, J., Monfils, M. H., and LeDoux, J. E. (2010). Fear and safety learning differentially affect synapse size and dendritic translation in the lateral amygdala. Proc. Natl. Acad. Sci. U.S.A. 107, 9418-9423. doi: 10.1073/ pnas.0913384107

Ostroff, L. E., Fiala, J. C., Allwardt, B., and Harris, K. M. (2002). Polyribosomes redistribute from dendritic shafts into spines with enlarged synapses during LTP in developing rat hippocampal slices. Neuron 35, 535-545. doi: 10.1016/S08966273(02)00785-7

Perez-Cruz, C., Simon, M., Czéh, B., Flügge, G., and Fuchs, E. (2009a). Hemispheric differences in basilar dendrites and spines of pyramidal neurons in the rat prelimbic cortex: activity- and stress-induced changes. Eur. J. Neurosci. 29, 738-747. doi: 10.1111/j.1460-9568.2009.06622.x

Perez-Cruz, C., Simon, M., Flügge, G., Fuchs, E., and Czéh, B. (2009b). Diurnal rhythm and stress regulate dendritic architecture and spine density of pyramidal neurons in the rat infralimbic cortex. Behav. Brain Res. 205, 406-413. doi: 10.1016/j.bbr.2009.07.021

Peters, A., and Kaiserman-Abramof, I. R. (1970). The small pyramidal neuron of the rat cerebral cortex. The perikaryon, dendrites and spines. Am. J. Anat. 127, 321-355. doi: 10.1002/aja.1001270402

Pozo, K., and Goda, Y. (2010). Unraveling mechanisms of homeostatic synaptic plasticity. Neuron 66, 337-351. doi: 10.1016/j.neuron.2010.04.028

Pyza, E., and Meinertzhagen, I. A. (1993). Daily and circadian rhythms of synaptic frequency in the first visual neuropile of the housefly's (Musca domestica L.) optic lobe. Proc. Biol. Sci. 254, 97-105. doi: 10.1098/rspb.1993. 0133

Pyza, E., and Meinertzhagen, I. A. (1999). Daily rhythmic changes of cell size and shape in the first optic neuropil in Drosophila melanogaster. J. Neurobiol. 40, 77-88. doi: 10.1002/(sici)1097-4695(199907)40:1<77::aid-neu7>3.0.co;2-0

Raven, F., Van der Zee, E. A., Meerlo, P., and Havekes, R. (2018). The role of sleep in regulating structural plasticity and synaptic strength: implications for memory 
and cognitive function. Sleep Med. Rev. 39, 3-11. doi: 10.1016/j.smrv.2017.05. 002

Ruiz, S., Ferreiro, M. J., Menhert, K. I., Casanova, G., Olivera, A., and Cantera, R. (2013). Rhythmic changes in synapse numbers in Drosophila melanogaster motor terminals. PLoS One 8:e67161. doi: 10.1371/journal.pone.0067161

Segal, M. (2010). Dendritic spines, synaptic plasticity and neuronal survival: activity shapes dendritic spines to enhance neuronal viability. Eur. J. Neurosci. 31, 2178-2184. doi: 10.1111/j.1460-9568.2010.07270.x

Sorra, K. E., and Harris, K. M. (2000). Overview on the structure, composition, function, development, and plasticity of hippocampal dendritic spines. Hippocampus 10, 501-511. doi: 10.1002/1098-1063(2000)10:5<501::AIDHIPO1>3.0.CO;2- T

Spacek, J., and Harris, K. M. (1997). Three-dimensional organization of smooth endoplasmic reticulum in hippocampal CA1 dendrites and dendritic spines of the immature and mature rat. J. Neurosci. 17, 190-203. doi: 10.1523/ JNEUROSCI.17-01-00190.1997

Tønnesen, J., Katona, G., Rózsa, B., and Nägerl, U. V. (2014). Spine neck plasticity regulates compartmentalization of synapses. Nat. Neurosci. 17, 678-685. doi: $10.1038 / \mathrm{nn} .3682$

Tønnesen, J., and Nägerl, U. V. (2016). Dendritic spines as tunable regulators of synaptic signals. Front. Psychiatry 7:101. doi: 10.3389/fpsyt.2016.00101

Tononi, G., and Cirelli, C. (2006). Sleep function and synaptic homeostasis. Sleep Med. Rev. 10, 49-62. doi: 10.1016/j.smrv.2005.05.002

Turrigiano, G. G., and Nelson, S. B. (2000). Hebb and homeostasis in neuronal plasticity. Curr. Opin. Neurobiol. 10, 358-364. doi: 10.1016/S0959-4388(00) 00091-x

van der Zee, E. A. (2015). Synapses, spines and kinases in mammalian learning and memory, and the impact of aging. Neurosci. Biobehav. Rev. 50, 77-85. doi: 10.1016/j.neubiorev.2014.06.012
Vlachos, A., Korkotian, E., Schonfeld, E., Copanaki, E., Deller, T., and Segal, M. (2009). Synaptopodin regulates plasticity of dendritic spines in hippocampal neurons. J. Neurosci. 29, 1017-1033. doi: 10.1523/JNEUROSCI.5528-08.2009

Wickens, J. (1988). Electrically coupled but chemically isolated synapses: dendritic spines and calcium in a rule for synaptic modification. Prog. Neurobiol. 31, 507-528. doi: 10.1016/0301-0082(88)90013-5

Woźnicka, O., Görlich, A., Sigrist, S., and Pyza, E. (2015). BRP-170 and BRP190 isoforms of Bruchpilot protein differentially contribute to the frequency of synapses and synaptic circadian plasticity in the visual system of Drosophila. Front. Cell. Neurosci. 9:238. doi: 10.3389/fncel.2015. 00238

Yang, G., and Gan, W. B. (2012). Sleep contributes to dendritic spine formation and elimination in the developing mouse somatosensory cortex. Dev. Neurobiol. 72, 1391-1398. doi: 10.1002/dneu.20996

Yuste, R., Majewska, A., and Holthoff, K. (2000). From form to function: calcium compartmentalization in dendritic spines. Nat. Neurosci. 3, 653-659. doi: 10. $1038 / 76609$

Conflict of Interest: The authors declare that the research was conducted in the absence of any commercial or financial relationships that could be construed as a potential conflict of interest.

Copyright $\odot 2020$ Jasinska, Woznicka, Jasek-Gajda, Lis, Pyza and Litwin. This is an open-access article distributed under the terms of the Creative Commons Attribution License (CC BY). The use, distribution or reproduction in other forums is permitted, provided the original author(s) and the copyright owner(s) are credited and that the original publication in this journal is cited, in accordance with accepted academic practice. No use, distribution or reproduction is permitted which does not comply with these terms. 